\title{
CIENCIA Y SOCIEDAD: NUEVAS INTERACCIONES EN EL UNIVERSO DIGITAL. HACIA UNA NUEVA DISCIPLINA ACADÉMICA PARA SU ESTUDIO
}

\author{
SCIENCE AND SOCIETY: NEW CONNECTIONS IN THE DIGITAL WORLD. A NEW \\ SCHOLAR FIELD TO BE RESEARCHED
}

\author{
LOURDES LÓPEZ-PÉREZ \& MARÍA DOLORES OLVERA-LOBO \\ lourdes.lpez@gmail.com - molvera@ugr.es \\ Universidad de Granada
}

\author{
RECIBIDO: 23/04/2018 \\ ACEPTADO: $17 / 07 / 2018$
}

\begin{abstract}
Resumen: La relación entre ciencia y sociedad ha cambiado radicalmente en los últimos 30 años desde el denominado modelo de déficit cognitivo (centrado en la falta de cultura científica de los ciudadanos) hasta la participación del público en la ciencia.

Una transformación impulsada por la irrupción de Internet que, no sólo ha favorecido un papel más activo de la sociedad en el desarrollo científico, sino que también ha generado un nuevo escenario de estudio centrado en el análisis de la democratización del proceso científico al amparo del universo digital y su impacto social y cultural.

Aunque aún es incipiente, ya existe una corriente académica que ha puesto el foco de atención en este nuevo campo de investigación humanística.

Estos autores apuntan, entre sus conclusiones, que el acceso abierto y la participación pública que posibilitan las herramientas de la Web 2.0 apoyan la socialización del proceso científico y contribuyen al desarrollo de una investigación e innovación responsable. En el presente trabajo se reivindica la importancia de desarrollar un marco teórico desde las ciencias sociales y humanidades digitales que permita analizar tanto el papel de Internet en el impulso de la RRI, como la calidad, efectividad y características de las interacciones digitales entre ciencia y sociedad.
\end{abstract}

Palabras clave: Comunicación pública de la ciencia, Web 2.0, participación del publico en la ciencia, investigación e innovación responsable, Internet

\begin{abstract}
The relation between science and society has radically changed in the last 30 years from the deficit model - in which the general public is defined negatively due to its lack of knowledge to the participative model. A transformation encouraged by the Internet irruption, which not only has improved a better role of society in scientific development but also it has created a new research field focused on the analysis of democratization of scientific process and its social and cultural impact.

However, this research area is yet emerging, it just exists a strong academic framework aiming attention at this new field of humanistic research.

These scholars indicate, among their conclusions, that open access and public engagement in science enhanced by web 2.0 tools promote the socialization of the scientific process and contribute to the consolidation of responsible research and innovation. The present article claims for the importance to develop a new framework from social sciences and digital humanities to study the Internet impact on the implementation of RRI and to analyze the quality, the effectivity and the characteristics of the digital connections between science and society.
\end{abstract}


Key words: Science communication, Web 2.0, public engagement in science, responsible research and innovation, Internet

\section{Introducción}

La ciencia definida como "elitista" por autores como Brown (2016) ha ganado una nueva humildad gracias a los diferentes canales de comunicación que posibilita la Web tales como las redes sociales, las wikis o los blogs (Brown, 2016). Ciertamente, las tecnologías digitales han transformado la esfera pública, la cual ya no consiste en un espacio físico al que acude el público sino que se trata más bien múltiples espacios virtuales que promueven la conversación y la participación de todos los agentes implicados en la investigación (Grand et al., 2016).

En este sentido, Internet ha favorecido el papel activo de los ciudadanos, quienes a través de este canal aprenden, evalúan, comparten, participan y deciden sobre el proceso de investigación científica (Brossard, 2013). De esta manera, el uso de los medios digitales en el proceso científico crea nuevos ecosistemas de investigación y cambia las prácticas de participación (Grand et al., 2016; Weilgod y Treise, 2004). Desde el punto de vista sociológico, puede decirse que la Web 2.0 ha promovido una sociedad más informada y con más conciencia sobre el conocimiento científico.

En este contexto, el impacto social de la comunicación pública de la ciencia a través de la red ha crecido exponencialmente en la última década tal y como demuestran los datos de la última encuesta de Percepción Social de la Ciencia y la Tecnología (Fundación Española de la Ciencia y la Tecnología, 2017). Este estudio pone de manifiesto que un $82,1 \%$ de jóvenes de entre 15 y 24 años, y un $77,5 \%$ de los que tienen entre 25 a 34 años, recurren a Internet para informarse sobre ciencia.

Por tanto, es una realidad que los nuevos canales de comunicación digitales están transformando las interacciones entre ciencia y sociedad. Esto provoca que desde el ámbito académico surja la necesidad de fijar el foco de atención, y la investigación, en la comprensión de este nuevo escenario. En países como Reino Unido y Estados Unidos son muchos los académicos de la comunicación de la ciencia que han empezado a establecer su objeto de estudio en el espacio digital, pero en nuestro ámbito nacional las iniciativas en este sentido son muy incipientes todavía.

En el presente artículo se subraya la importancia de desarrollar un marco teórico desde las ciencias sociales y las humanidades digitales que permita 
analizar, tanto el papel de Internet en el impulso de la participación del público en la ciencia, como la calidad, efectividad y características de las interacciones digitales entre ciencia y sociedad desde múltiples perspectivas.

Para ello, en los siguientes apartados se describe la evolución de la relación entre la ciencia y la sociedad -desde el modelo de déficit cognitivo al actual modelo de participación del público en la ciencia-, se expone el impacto de la Web 2.0 en la comunicación entre científicos y ciudadanos a través de la literatura científica, y se presentan algunas de las líneas de investigación actuales que deberían tener continuidad de futuro. La realidad construida al amparo del universo digital es tan compleja que, como concluimos en este trabajo, requiere investigaciones interdisciplinares que incorporen campos del conocimiento implicados en las humanidades y ciencias sociales digitales como son la educación, el periodismo, la ética, la filosofía, la comunicación, la sociología o la lingüística, entre otros.

\section{De la alfabetización a la participación pública en la ciencia}

En el debate académico sobre la relación entre ciencia y sociedad se pueden distinguir tres etapas marcadas por el papel jugado por los protagonistas de este binomio, esto es, la ciencia y la sociedad (Alix et al., 2008; Olvera-Lobo y López-Pérez, 2014b; López-Pérez y Olvera-Lobo, 2015b): i) alfabetización científica y comprensión pública de la ciencia (Whitey, 1959, Shen, 1975; Thomas y Kindo, 1978; Miller, 1983; Koelsche, 1965; Bodmer, 1985), ii) diálogo o comunicación pública de la ciencia (Royal Society, 2000; Michael, 2002; Pardo y Calvo, 2002; Dierkens y Von Grote, 2003; Hanssen et al., 2003; Winter, 2004; Davies, 2011 y Stilgoe, Lock y Wilsdom, 2014) y iii) participación pública en la ciencia (Rowe y Frewer, 2005; Hagendijk e Irwin, 2006; Alix et al., 2008; Bonney et al., 2009; National Co-ordinating Centre for Public's Engagement, 2010; Árnason, 2012; Irwin, Jensen y Jones, 2012; Stilgoe, Lock y Wilsdom, 2014; Rarn, Mejlgaard y Rask, 2014; Klüver et al., 2014; Rask et al., 2016).

La primera etapa anteriormente mencionada, también denominada modelo de déficit cognitivo, pone especial énfasis en el análisis del interés y de la comprensión de la ciencia por parte de los ciudadanos. Defiende una visión positivista estableciendo que solo la falta de conocimiento genera una ausencia de apoyo social a los avances científicos. De este modo, culpa de las dificultades de la relación entre ciencia y sociedad al desconocimiento del público (Michael, 2002). Los ciudadanos son imaginados como ignorantes y hostiles (Alix et al., 
2008) y los actores responsables de revertir esta situación son los propios ciudadanos, los políticos y los educadores, exonerando a los científicos de cualquier obligación a la acción.

La evolución de los estudios de comunicación pública de la ciencia y el desarrollo de informes como el Science and Technology Third Report (Royal Society, 2000) generaron una corriente de crítica a este modelo considerado como retrógrado y despectivo, además de responsable de la falta de confianza del público a la ciencia (Royal Society, 2000) e impulsan el concepto de diálogo para referirse a la relación ideal entre ciencia y sociedad (Royal Society, 2000; Michael, 2002; Pardo y Calvo, 2002; Dierkens y Von Grote, 2003; Hanssen et al., 2003; Winter, 2004; Davis, 2011 y Stilgoe, Lock y Wilsdom, 2014). En esta segunda etapa, se pasa de una visión elitista de la ciencia (Brown 2016) a una idea más democrática del proceso científico en el que productores y usuarios comparten e intercambian conocimientos, ideas, valores, actitudes y creencias entre organizaciones, científicos, público y políticos (Jackson, Barbagallo y Haste, 2005; Davies, 2011). En esta misma línea se sitúan otros autores (Burns, O'Conner, y Stocklmayer, 2003; Felt, 2003; Van Dijk, 2003; Rowe y Frewer, 2005; Olvera-Lobo y López Pérez, 2014b; López-Pérez y Olvera Lobo, 2015b) al acuñar el término comunicación pública de la ciencia.

Con el desarrollo de avances tecnológicos de gran impacto social que conllevan la implicación de cuestiones éticas asociadas a la investigación se da un paso más en la relación entre ciencia y sociedad, centrándose en la actualidad en la participación pública en el proceso científico, con lo que nos adentramos en la tercera fase del debate académico sobre la relación entre ciencia y sociedad.

En efecto, en la última década y, coincidiendo con la generalización del uso de la Web 2.0, la conceptualización de la comunicación pública de la ciencia y su foco de estudio han experimentado cambios suscitados por la transformación de la relación ciencia y sociedad, generada, en gran medida, por el nuevo espacio conversacional que ofrece Internet (Weilgod y Treise, 2004; Yang, Kong y Jhonson, 2010; Saffer, Sommerfeldt y Taylor, 2013; Grand et al, 2016; Brown, 2016; Su, Akin y Brossard et al., 2017; Pitrelli, 2017).

Así, este nuevo ímpetu por potenciar el diálogo entre científicos y ciudadanos se ha reflejado en la evolución del interés académico ya que, éste ha mudado desde el nivel de conocimiento científico adquirido por la sociedad hacia el nivel de implicación de los ciudadanos en el proceso científico; desde la comunicación unidireccional de la ciencia, al diálogo entre científicos y ciudadanos; desde el binomio ciencia y sociedad, a la ciencia con y para la sociedad (Bucchi y Neresini, 2008). 
Una nueva realidad que se ha reflejado en la literatura científica con un cambio de paradigma que ha evolucionado desde el modelo de déficit cognitivo -en el que el público es definido negativamente por su falta de conocimientoshasta el modelo participativo -en el que el público es invitado a formar parte de la empresa científica-.

De este modo, la participación del público en la ciencia es el paradigma actualmente consolidado en el ámbito académico. Este modelo, que va más allá de la comunicación unidireccional involucrando a los ciudadanos en el desarrollo de la $\mathrm{I}+\mathrm{D}+\mathrm{i}$, ha adquirido nuevas dimensiones al integrarse como uno de los elementos clave dentro de la investigación e innovación responsables (RRI), un concepto que está penetrando en las políticas científicas europeas a través del programa Horizon 2020 (Owen, Macnaghten y Stilgoe, 2012).

Aunque el concepto de participación pública en la ciencia aparece en la literatura científica hace más de una década, aún no existe consenso sobre qué supone esta implicación de los ciudadanos en la ciencia. Investigadores como Rowe y Frewer (2005) consideran participación del público en la ciencia como una combinación entre la comunicación, la consulta y la intervención pública en el marco de la investigación y la innovación. Por su parte, Rarn, Mejlgaard y Rask (2014) parten de la categorización de Rowe y Frewer (2005) y plantean una clasificación que integra las diferentes iniciativas de participación del público como comunicación pública, activismo público, consulta pública y deliberación pública.

Por su parte, Bucchi y Neresini (2008) categorizan la participación del público en la ciencia como normalizada (encuestas de público, evaluación participativa de tecnología o iniciativas democráticas de consenso) y no normalizada o espontánea (protestas locales, movimientos sociales, investigación realizada por la comunidad o asociaciones de pacientes). Otros académicos como Bonney et al. (2009) definen la participación del público ciñéndola a las diferentes etapas del proceso de investigación e innovación en las que los ciudadanos pueden ser partícipes: $i$ ) elegir o definir las preguntas del estudio; $i$ ) buscar información y recursos; iii) desarrollar hipótesis y posibles respuestas a las mismas; iv) recopilar datos; v) analizar datos; vi) interpretar los datos y establecer conclusiones; vii) difundir las conclusiones; y viii) discusión de resultados y generación de nuevas preguntas. Así, dependiendo del grado de implicación de los ciudadanos, describen tres formas en las que los éstos pueden implicarse en el proceso de I+D+i, a saber, proyectos de contribución, proyectos de colaboración y proyectos de co-creación.

Para Klüver et al. (2014) la mayoría de las actividades de participación pública se basan en la implicación de los ciudadanos en las fases del proceso 
científico relativas a: i) establecimiento de la agenda de investigación e innovación; ii) supervisión y evaluación de la investigación y la innovación; iii) implicación activa en la investigación y financiación de la misma; $i v$ ) aportación de conocimiento específico sobre su entorno; v) recopilación de datos; y vi) divulgación de los resultados de investigación.

Como se ha indicado, la irrupción de Internet favorece la implicación de los ciudadanos en el proceso científico y facilita el desarrollo de este modelo participativo si bien las evidencias científicas sobre su impacto son aún escasas.

\section{Internet como canal para la participación del público en la ciencia}

Hasta 1998, los académicos no centran su foco de estudio en Internet como canal para la difusión del conocimiento científico (Eveland y Dunwoody, 1998; Byrne et al, 2002). Su capacidad para generar debate y discusiones sobre temas científicos es lo que ha alentado a autores, ingleses y americanos principalmente, (Rogers y Marres, 2000; Triunfol, 2004; Delborne et al, 2011) a fijar la mirada en esta fuente inagotable de conocimiento para las multitudes (Shirky, 2010).

Estudiosos de la comunicación pública de la ciencia como Weilgod (2001) aseguran que Internet ha cambiado radicalmente las relaciones entre los actores de la comunicación de la ciencia por varias razones. Por un lado, la Web permite a los científicos y a sus organizaciones comunicarse directamente con sus audiencias. Además, elimina las restricciones de tiempo y espacio inherentes a los medios de comunicación. Asimismo, combina la capacidad de profundización de la prensa escrita con las posibilidades de interacción y de comunicación con los usuarios que ofrece la Web 2.0. Y, por último, facilita la comunicación instantánea de uno a uno, de uno a muchos, de muchos a uno y de muchos a muchos.

Y es que Internet ha devuelto la ciencia a la esfera pública. Después de más de un siglo de aislamiento, los científicos vuelven a estar ante el público. Esta vez, ya no se trata de meros espectadores que asisten a la representación de la ciencia, sino de agentes activos que aprenden, evalúan, comparten, participan y deciden (Brossard, 2013).

La Web social ha posibilitado la desintermediación de la comunicación pública de la ciencia y ha recuperado el ideal de la democratización del conocimiento, transformando la inaccesible Torre de Marfil de los científicos en un ágora abierta a los ciudadanos (Baron, 2010; Olvera-Lobo y López- Pérez, 2013, 2014a, 2014b; López-Pérez y Olvera-lobo, 2015a, 2015b, 2016a, 2016b) 
Valorados como un canal clave en el aprendizaje informal de la ciencia (Eveland y Dunwoody, 1998; Lederbogen y Trebbe, 2003; Weilgod y Treise, 2004), los sitios web científicos pueden transformar el proceso de comprensión, desde la memorización pasiva a la implicación activa (Weilgod y Treise, 2004).

De esta manera, Internet se presenta como un medio para incrementar la urgente necesidad de diálogo entre científicos y público (Lederbogen y Trebbe, 2003) y cuenta con capacidad para eliminar la creencia en las habilidades mágicas de los científicos, al tiempo que se consigue un mayor apoyo del público a la investigación gracias al conocimiento y confianza mutua. Los sitios web de ciencia constituyen por tanto una importante herramienta para frenar la incultura científica, promover actitudes positivas hacia la ciencia y fomentar vocaciones científicas (Ebersol, 2000). Indiscutiblemente, Internet ha hecho mucho más permeable la frontera entre la comunicación profesional y la conversación con el público, facilitando el acceso de la sociedad a una parcela que antes era privada y favoreciendo la "desintermediación" de la ciencia (Trench, 2008).

En este contexto, los medios de comunicación ya no son los únicos responsables de la cultura y educación científica de los ciudadanos. Ahora los investigadores y las instituciones públicas tienen la responsabilidad de llevar a la esfera pública la conversación sobre la ciencia (Batts, Anthis y Smith, 2008; Olvera-Lobo y López-Pérez, 2013a, 2013b, 2014a, 2014b; López-Pérez y Olvera-Lobo, 2015a, 2015c).

Por último, es reseñable el hecho de que, hasta no hace mucho tiempo, la mayor parte de la literatura científica relacionada se limita, principalmente, a analizar los blogs, una de las herramientas de la Web 2.0 considerada como uno de los mejores canales para la comunicación pública de la ciencia (Trench, 2008), no sólo por su potencial para presentar los resultados científicos a la sociedad (Lapointe y Drouin, 2007; Wilkins, 2008; Kouper, 2010; Colson, 2011), sino también por su capacidad para cambiar radicalmente el proceso de producción científica (Trench, 2008; Batts, Anthis y Smith, 2008; Mahrt y Puschmann, 2013).

\section{Comunicación digital de la ciencia: objeto de estudio interdisciplinar}

Para disciplinas como las humanidades y las ciencias sociales, la Web 2.0 se ha convertido en un importante objeto de estudio donde analizar la realidad generada al amparo de este universo digital. La comunicación pública de la ciencia es uno de los ámbitos académicos donde el interés científico por la dimensión digital empieza a cobrar importancia en áreas que van desde el 
análisis de controversias científicas a la ciencia ciudadana, o de la definición de nuevas formas y prácticas para el periodismo científico a la ciencia abierta ( $\mathrm{Su}$, Akin y Brossard, 2017; Pitrelli, 2017; Grand et al, 2016; Olsson, 2016; LópezPérez y Olvera-Lobo, 2016a, 2016b, 2015; Olvera-Lobo y López-Pérez, 2014, 2013a, 2013b; Mahrt y Puschmann, 2013; Colson, 2011; Kouper, 2010).

El impacto de la comunicación pública de la ciencia a través de la red en la producción científica es otra de las líneas de investigación que ha surgido en los últimos años. Shuai, Pepe y Bolen (2012) concluyeron que la comunicación de un paper a través de Twitter puede incrementar hasta en 11 veces las posibilidades de que un artículo sea citado. Por su parte, Jensen et al. (2008) confirmaron que los científicos con mayor participación en actividades de divulgación científica habían publicado más artículos científicos y eran más citados en un año que sus colegas que no lo habían hecho.

El nuevo rol de los científicos en la relación con la sociedad también ha sido foco de trabajos como el de Grand et al. (2016). Estos investigadores analizaron la forma en que los investigadores conectan con los diferentes agentes sociales a través de los medios digitales para crear, compartir y representar el conocimiento conjuntamente. Entre sus conclusiones apuntaron que el acceso abierto y la participación facilitados por las herramientas de la Web 2.0 fomentan la implicación del público en el proceso científico, contribuyendo a la socialización de la investigación.

Por su parte, Lee y Van Dyke (2015) establecieron su interés en el impacto de la red sobre las relaciones públicas de las instituciones científicas. Sus resultados señalan de qué manera la Web 2.0 permite a las instituciones establecer un diálogo con su público, cultivar una mejor relación con los diferentes agentes sociales y activar la participación online y offline de la audiencia.

Desde el punto de vista de la promoción de la cultura científica, académicos como Schefeule (2014) afirman que el mecanismo clave detrás del aprendizaje informal de las ciencias es la comunicación bidireccional entre las instituciones y el público. Otros estudiosos como (Kwok y Yu, 2013; Saffer, Sommerfeldt y Taylor, 2013 y Yang et al., 2013) presentan además evidencias científicas que respaldan que la interactividad promovida por las instituciones a través de las redes sociales influye positivamente en las actitudes, percepciones y conductas de las sociedad.

\section{Conclusiones}

La comunicación pública de la ciencia digital está irrumpiendo como una nueva disciplina académica con múltiples focos de estudio que deben abordarse desde 
una perspectiva multidisciplinar. A las líneas de investigación surgidas en los últimos años, principalmente en el ámbito internacional, deben sumarse otras de importante interés como el análisis del contenido de las interacciones entre ciencia y sociedad o el estudio de la viralidad de noticias científicas falsas que pueden afectar a aspectos de relevancia social como la salud.

La generación de nuevas fuentes de información periodística como los blogs o Twitter, el uso de las nuevas herramientas de la Web 2.0 para implicar a los ciudadanos en el proceso científico o el cambio de relación entre científicos y periodistas son otros de los aspectos que deben ser abordados desde las ciencias sociales digitales. Las ventajas y desventajas sociales de la ciencia abierta, el fomento de vocaciones científicas o el impulso de la ciencia ciudadana a través de las plataformas digitales también han de convertirse en objeto de estudio en los últimos años.

Como se ha expuesto a través de esta breve revisión de la literatura científica, la comunicación pública de la ciencia digital es un campo emergente que requiere un marco teórico y un cuerpo académico que necesita consolidarse en España. Deseamos que esta aportación sirva de acicate para el impulso de nuevos trabajos académicos que ayuden a comprender cómo Internet está cambiando las interacciones entre ciencia y sociedad.

\section{Bibliografía}

AliX, J. ET AL. (2008). Public Engagement in Science across the European research Area. En: Report of the science in society session. Brussels: Public Engagement in Science European Communities, (DOI: http://dx.doi.org/10.2777/20800)

ÁrnASON, V. (2012). "Scientific Citizenship in a Democratic Society". Public Understanding of Science 22 (8): pp. 927-940 (DOI: http://dx.doi.org/10.1177/0963662512449598)

BARON, N. (2010). "Escape from the ivory tower". Washington: Island Press ISBN: 9781597266635

BAtTs, S., ANTHIS, N. Y SMITH, T. (2008). "Advancing Science through Conversations: Bridging the Gap between Blogs and the Academy". $\begin{array}{lllll}P L O S & \text { Biology } & \text { (6) } & 9 & \text { (DOI: }\end{array}$ http://dx.doi.org/10.1371/journal.pbio.0060240)

BYRNE, P. ET AL (2002). "Increasing public understanding of transgenic crops through the World Wide Web". Public Understanding of Science 11(3) pp. 293-304 (DOI: http://dx.doi.org/10.1088/0963-6625/11/3/306) 
Bodmer, W. (1985). The Public Understanding of Science. London: The Royal Society. Recuperado de: https://royalsociety.org/ /media/Royal_Society_Content/policy/publicat ions/1985/10700.pdf

BONNEY, R. ET AL (2009). Public participation in scientific research: Defining the field and assessing its potential for informal science education. A caisi inquiry group report. Washington DC.: Center for Advancement of Informal Science Education.

BRown D. (2016). Access to scientific research. Challenges facing communications in STM. Berlin: De Gruyter.

BROSSARD, D. (2013). New media landscapes and the science information consumer. PNAS 10 (3) DOI: https://doi.org/10.1073/pnas.1212744110

BucchI, M. AND NeresinI, F. (2008). "Science and Public Participation". En: The handbook of science and technology studies. Ed. by Felt, U.; Fouché, R.; Miller, C and Smith-Doerr, L. (pp. 449-472). MIT-press ISBN: 9780262035682

Burns, T., O'CONNER, D. Y StOcKLmaYer, S. (2003). "Science Communication: A Contemporary Definition". Public Understanding of Science $\quad 12 \quad$ (2): $183-202$ (http://dx.doi.org/10.1177/09636625030122004)

COLSON, V. (2011). "Science blogs as competing channels for the dissemination of science news". Journalism (12) 7 pp. 849-889 DOI: http://dx.doi.org/10.1177/1464884911412834)

DELBORNE, J. ET AL (2011). "Virtual deliberation? Prospects and challenges for integrating the Internet in consensus conferences". Public Understanding of Science 20(3) pp. 367-384 (DOI: HTTP://DX.DOI.ORG/10.1177/0963662509347138)

DIERKENS, M. Y VON GROTE, C. (2003). Between Understanding and Trust: The Public, Science and Technology. London: Routledge.

DAVIES, S. (2011). "The Rules of Engagement: Power and Interaction in Dialogue Events". Public Understanding of Science 22 (65) (DOI: http://dx.doi.org/10.1177/0963662511399685)

EBERSOL, S. (2000). "Uses and Gratifications of the Web among Students". Journal of Computer-Mediated Communication (6)1 pp. 161-182 DOI: http://dx.doi.org/10.1111/j.1083-6101.2000.tb00111.x)

EVELAND, W. Y DUNWOODY, S. (1998). "Users and navigation patterns of a science World Wide Web site for the public". Public Understanding of Science 7 (4) pp. 285-311 (DOI: http://dx.doi.org/ 10.1088/0963$6625 / 7 / 4 / 003)$ 
Felt, U. (2003). "Why Should the Public 'Understand' Science? A Historical Perspective on Aspects of the Public Understanding of Science". En Dierkens, M. and Von Grote, C. Between Understanding and Trust: The Public, Science and Technology. London: Routledge.

FUNDACIÓN ESPAÑOLA PARA LA CIENCIA Y LA TECNOLOGÍA (2017). VIII Encuesta de Percepción Social de la Ciencia y la Tecnología 2016. https://www.fecyt.es/es/noticia/crece-el-interes-de-las-mujeres-por-laciencia-y-la-tecnologia

GRAND A ET AL. (2016). "We muddle our way through: shared and distributed expertise in digital engagement with research". Journal Science Communication: 15(4). Recuperado de: https://jcom.sissa.it/archive/15/04/JCOM_1504_2016_A05

HAGENDIJK, R. E IRWIN, A. (2006). "Public deliberation and governance: engaging with science and technology in contemporary Europe". Minerva, 2006; 44: pp.: 167-184 8DOI: http://dx.doi.org/10.1007/s11024-006-0012-x)

HANSSEN ET AL. (2003). Wetenschapsvoorlichting profetie of professie:een confrontatie tussen communicatietheorie en voorlichtingspraktijk. Amsterdam: Report Stiching WeTeN.

IRWIN, A., JENSEN, T. Y JONES, K. (2012). "The good, the bad and the perfect: Criticizing engagement practice". Social Studies of Science 43 (1): pp.:118-135 (DOI: http://dx.doi.org/10.1177/0306312712462461)

JaCKSON, R., BARBagallo, F. Y HASTE, H. (2005). "Strengths of public dialogue on science-related issues". Critical Review of International Social and Political Philosophy 8 (3): pp. 349-358 (DOI: http://dx.doi.org/10.1080/13698230500187227)

JENSEN ET AL (2008). Scientists who engage with society perform better academically. Science and public policy (7) 35 pp. 527-541

KlÜVer, L. ET AL (2014). Public Engagement in R\&I processes. Promises and demands. Engaging Society in Horizon 2020. Disponible en: http://engage2020.eu/media/Engage2020-Policy-Brief-Issue2_final.pdf

Koelsche, C. (1965). "Scientific literacy as Related to the Media of Mass Communication”. School Science and Mathematics 65 (8): pp.719-725 (http://dx.doi.org/10.1111/j.1949-8594.1965.tb13564.x)

KOUPER, I. (2010). Science blogs and public engagement with science: practices, challenges and opportunities. Journal of Science Communication (69) 1 
KwoK, L., \& YU, B. (2013). Spreading social media messages on Facebook: An analysis of the restaurant industry. Cornell Hospitality Quarterly, 54(1), 84-94.

LAPOINTE, P. y Drouin, J. (2007). Science on Blogue. Quebec: Multimundes.

Lederbogen, U. y Trebbe, J. (2003). "Promoting Science on the Web. Public Relations for Scientific Organizations. Results of a Content Analysis". Science Communication (24) 3 pp. 333-352 DOI: http://dx.doi.org/10.1177/1075547002250299

LeE, N., Y VANDYKe, M. S. (2015). "Set it and forget it: The one-way use of social media by government agencies communicating science". Science Communication, 37(4), 533-541.

LÓPEZ-PÉREZ, L. Y OLVERA-LOBO M. (2015 a). "Comunicación de la ciencia 2.0 en España: El papel de los centros públicos de investigación y de medios digitales". Revista mediterránea de comunicación, 6(2). (DOI: http://dx.doi.org/10.14198/MEDCOM2015.6.2.08)

LÓPEZ-PÉREZ, L. Y OLVERA-LOBO, M. (2015 b). "De la alfabetización científica a la comunicación pública de la ciencia en España”. En: López-Ornelas, M. y Mateos, C. La comunicación científica, una perspectiva universitaria. Tenerife: Sociedad Latina de Comunicación Social, $2015 b$ ISBN: 13:978-84-16458-24-0

DOI: http://dx.doi.org/10.4185/cac93.

LÓPEZ-PÉREZ L. Y OLVERA-LOBO MD (2016 a). "Social media as channels for the public communication of science. The case of Spanish research centers and public universities". En: Knautz, Kathrin; Baran, Katsiaryna S. (eds.). Facets of Facebook. Users and use. De Gruyter House. ISBN: 9783110418163

LÓPEZ-PÉREZ, L. Y OlverA-Lobo, M. (2016 b). "La Web 2.0 para la Comunicación pública de la ciencia. El caso de los centros de investigación y universidades públicas españolas". El Profesional de la Información 25 (3): pp: 441-448 eISSN: 1699-2407

MAHRT, M. Y PUSCHMANN, C. (2013). Science blogging: an exploratory study of motives, styles, and audience reactions. Journal of science Communication (13) 3 pp. 1-16

Michael, M. (2002). "Comprehension, Apprehension, Prehension: Heterogeneity and the Public Understanding of Science". Science, Technology \& Human Values 27 (3) pp.357-78 (DOI: http://dx.doi.org/10.1177/016224390202700302) 
MiLleR, J. (1983). "Scientific Literacy: A Conceptual and Empirical Review". Daedalus 112(2): pp. 29-48. Recuperado de: http://www.jstor.org/stable/20024852 (18-07-2014)

NAtional Co-Ordinating Centre For Public Engagement (2010). What is Public Engagement. Recuperado de: https://www.publicengagement.ac.uk/explore-it/what-publicengagement (13-06-2014)

OLVERA-LOBO, M. Y LÓPEZ-PÉREZ, L. (2013). “The role of public universities and the primary digital national newspapers in the dissemination of Spanish science through the Internet and Web 2.0". En: TEEM '13 Procs of the First intl conf on technological ecosystem for enhancing multiculturality. New York: ACM pp. 191-196. ISBN: 97814503 23451 (DOI: http://dx.doi.org/10.1145/2536536.2536565)

OLVERA-LOBO, M. Y LÓPEZ-PÉREZ, L. (2014 a). "Science communication 2.0: The situation of Spain through its public universities and the most widely-circulated online newspapers". Information resources management journal 27(3), pp. 42-58. http://dx.doi.org/10.4018/irmj.2014070104

OlVERA-LOBO, M. Y LÓPEZ-PÉREZ, L. (2014 b). "Relación ciencia-sociedad: evolución terminológica". En: Gonzálvez-Vallés, Juan-Enrique (coord.). Comunicación actual: Redes sociales y lo 2.0 y 3.0. Madrid: McGraw Hill. ISBN: 9788448197469

Owen, R., Macnaghten, P. AND Stilgoe, J. (2012). "Responsible research and innovation: From science in society to science for society, with society". Science and Public Policy 39 (6) pp: 751-760. DOI: https://doi.org/10.1093/scipol/scs093

PARDO, R. y CALVO, F. (2002). "Attitudes toward science among the European public: A methodological analysis". Public Understanding Science, 2002; 11(2): pp. 155-195 (DOI: http://dx.doi.org/10.1088/0963$6625 / 11 / 2 / 305)$

Pitrelli, N. (2017). "Big Data and digital methods in science communication research: opportunities, challenges and limits". Journal of Science Communication $16 \quad$ (02). Recuperado de: https://jcom.sissa.it/archive/16/02/JCOM_1602_2017_C01

Rarn, T., MejlgaARd, N. Y Rask, M. (2014). Public Engagement Innovation for Horizon 2020. Inventory of PE mechanisims and initiatives. Disponible en: www.PE2020.eu

RASK, M. ET AL (2016). Innovative Public Engagement. A conceptual model of public engagement in Dynamic and Responsible Governance of 
Research and Innovation European Union's Seventh Framework. Programme for research, technological development and demonstration. Disponible en: https://pe2020.eu/2016/05/26/innovativepublic-engagement-a-conceptual-model-of-pe/

ROYAL SOCIETY (2000). Science and Technology: Third Report. Recuperado de: http://www.publications.parliament.uk/pa/ld199900/ldselect/ldsctech/38 13801.htm (10-06-2014)

RoGers, R. Y MARRES, N. (2000). "Landscaping climate change: A mapping technique for understanding science and technology debates on the World Wide Web". Public Understanding of Science 9(2) pp. [141-163] (DOI: http://dx.doi.org/10.1088/0963-6625/9/2/304)

Rowe, G. Y FREWER, L. (2005). "A Typology of Public Engagement Mechanisms". Science, Technology, and Human Values 30 (2): pp. 251-90. (DOI: http://dx.doi.org/10.1177/0162243904271724)

SAFFer, A., SOMmerfeldT, E. AND TAYlOR, M. (2013). "The effects of organizational Twitter interactivity on organization-public relationships". Public Relations Review 36 (4), pp. 336-341 DOI: https://doi.org/10.1016/j.pubrev.2013.02.005

SCHEUFELE, D. A. (2014). "Science communication as political communication". Proceedings of the National Academy of Sciences, 111(Suppl. 4), 13585-13592.

SHEN, B. (1975). "Scientific Literacy and the Public Understanding of Science". Communication of Scientific Information, 63(3): pp. 44-52

SHIRKY, C. (2010). Cognitive Surplus: Creativity and Generosity in a Connected Age. New York: Penguin Press ISBN: 1594202532

ShuAi, X., PePE, A. Y Bolen, J. (2012). How the scientific community reacts to newly submitted preprints: Article downloads, Twitter mentions, and citation. PLoS ONE (7) 11 e47523 (DOI: http://dx.doi.org/10.1371/journal.pone.0047523)

Stilgoe, J., Lock, S. Y Wilsdon, J. (2014). "Why Should We Promote Public Engagement with Science?" Public Understanding of Science 23(1): pp. 4-15 (DOI: http://dx.doi.org/10.1177/0963662513518154)

SU, AKIN Y BROSSARD (2017). "Information-sharing and Community-Building: Exploring the Use of Twitter in Science Public Relations". Science Communication 39(5) pp. 569-597 DOI: 10.1177/1075547017734226

THOMAS, F. Y KINDO A. (1978). Towards Scientific Literacy. Tehran: International Institute for Adult Literacy Methods and Hulton Educational Publications. 
Trench, B. (2008). HandBook of Public Communication of Science and Technology. USA: Routledge ISBN-13: 978-0415386173

TRIUNFOL, M. (2004). "Dynamics of list-server discussion on genetically modified foods". Public Understanding of Science 13(2) pp. 155-175 (DOI: http://dx.doi.org/10.1177/0963662504044110)

VAN DiJCK, J. (2003). "After the "Two Cultures": Toward a "(Multi)cultural" Practice of Science Communication". Science Communication 25(2): pp. 177-190 (DOI: http://dx.doi.org/10.1177/1075547003259540)

WEILGOD, M. Y TREISE, D. (2004). "Attracting teen surfers to science web sites". Public understanding science 13(3) pp. 229-248 DOI: http://dx.doi.org/10.1177/0963662504045504

WhiteY S. (1959). "Public Opinion about science and scientists". Public Opinion Quarterly, 1959 23(3): pp. 382-388 (DOI: http://dx.doi.org/10.1086/266890)

WILKINS, J. (2008). The roles, reasons and restrictions of science blogs. Trends in Ecology \& Evolution (23) 8 pp. 411-413 DOI: http://dx.doi.org/10.1016/j.tree.2008.05.004

WINTER, E. (2004). "Public Communication of Science and Technology: German and European Perspectives". Science Communication 25(3) pp. 288-293 (DOI: http://dx.doi.org/10.1177/1075547003262665)

YANG, S., KANG M., AND JOHNSON, P. (2010). "Effects of narratives, openness to dialogic communication through organizational blogs". Communication research 37 (4) pp.: 473-497 DOI: 10.1177/0093650210362682 
